\section{Contribuições da análise comparada para um marco abrangente na avaliação de sistemas orientados pela atenção primária na América Latina}

\section{The contribution of comparative analysis to a comprehensive evaluation framework for primary care systems in Latin America}

Eleonor Minho Conill 1

Márcia Cristina Rodrigues Fausto 2

Lígia Giovanella 3

\begin{abstract}
Objectives: to demonstrate that the study of integration and coordination of care may reveal significant elements for the evaluation of primary health care policy systems (PHC).

Methods: a descriptive study of the forms and factors that facilitate or impede the integration of PHC, using comparative analysis of the reforms carried out in Latin America, by way of systematic review and analysis of documents.

Results: three types of integration were identified: selective primary care within maternal/child health programs, PHC as first contact care and municipal experiments with insurance-based systems. An explanatory model of the problem of fragmentation is developed, demonstrating the need to articulate the analysis of the macro, meso and microsocial levels for a comprehensive view of the performance of services.

Conclusions: services remain segmented and fragmented, even in the case of systems with a universal basis. The proposed model combines these two categories, thereby contributing to the selection of indicators for evaluation of public systems guided by this policy.

Key words Latin America, Primary health care,
\end{abstract} Health evaluation, Integrated health care systems
${ }^{1}$ Departamento de Saúde Pública. Universidade Federal de Santa Catarina. Rua Vento Sul, 306. Florianópolis, SC, Brasil. CEP: 88.063-070. E-mail: eleonorconill@yahoo.com.br 2,3 Escola Nacional de Saúde Pública. Fundação Oswaldo Cruz. Rio de Janeiro, RJ, Brasil.

\section{Resumo}

Objetivos: demonstrar que o estudo da integração e da coordenação do cuidado traz elementos importantes para a avaliação de sistemas orientados por uma política de atenção primária de saúde (APS).

Métodos: estudo descritivo das formas e fatores que facilitam ou dificultam a integração da APS, a partir da análise comparada de reformas realizadas na América Latina, através de revisão sistemática e análise de documentos.

Resultados: três modalidades de integração foram identificadas: atenção primária seletiva no interior de programas materno-infantis, APS como porta de entrada e eixo estruturante de um sistema público e experiências municipais em sistemas baseados em seguros. Um modelo explicativo da problemática da fragmentação é desenvolvido, mostrando a necessidade de articular a análise dos níveis macro, meso e microssocial para uma visão abrangente do desempenho dos serviços.

Conclusões: há persistência de segmentação com fragmentação dos serviços, mesmo nos casos de sistemas de base universal. O modelo proposto articula essas duas categorias, contribuindo na seleção de indicadores para avaliação de sistemas públicos orientados por essa política.

Palavras-chave América Latina, Atenção primária à saúde, Avaliação em Saúde, Prestação integrada de cuidados de saúde 


\section{Introdução}

Uma reflexão interessante que os estudos comparados possibilitaram circunscrever é a existência de uma dinâmica de convergência e divergência entre os sistemas contemporâneos de saúde com semelhanças no compartilhamento de alguns problemas e diferenças determinadas por especificidades de cada formação social. ${ }^{1}$

A segmentação e a fragmentação surgem como categorias centrais para a compreensão da problemática que é hoje comum aos sistemas profissionais e sua relação com a qualidade dos serviços. A segmentação relaciona-se com as garantias de proteção social, com o acesso, a equidade e a coesão social. Já a fragmentação diz respeito à organização e à prestação dos serviços, sua coordenação com reflexos na integralidade, continuidade dos cuidados, crescimento dos gastos e perda de eficiência.

$\mathrm{O}$ argumento deste texto é que o estudo da integração e da coordenação do cuidado traz elementos importantes para a avaliação do desempenho de sistemas orientados por uma política de atenção primária de saúde (APS). Tem como referencial um recorte, com enfoque na América Latina, de um estudo realizado para compreender os fatores que facilitam ou dificultam a integração da APS, a partir da análise comparada de reformas realizadas nesse continente e na União Européia. ${ }^{2}$

Após um período de reformas neoliberais, o novo milênio iniciou com um discurso enfatizando a importância da APS na organização de sistemas públicos. ${ }^{3}$ Mas, não há um entendimento uniforme a respeito dessa política e sua implementação acompanha a diversidade de sistemas de proteção social e de serviços de saúde atualmente existentes na região.

Haggerty et al. 4 estudaram a pertinência de utilizar na América Latina uma estratégia de avaliação da APS desenvolvida no Canadá através de um consenso obtido por experts em torno de um conjunto de dimensões e indicadores. Seis objetivos principais compõem a proposta canadense: acesso universal, qualidade técnica, aceitabilidade, porta de entrada, atenção integral e integrada, coordenação e planejamento. O grupo destacou a necessidade de conhecer os diversos significados da APS, sendo que os objetivos de coordenação, atenção integral e integrada não obtiveram um alto nível de consenso em função da existência de múltiplas interpretações, mostrando que um referencial para seu monitoramento e avaliação deverá levar em conta a diversidade de interpretações e das modalidades de sua implantação.

Quais as principais características das reformas em curso e que estratégias têm sido utilizadas para facilitar o papel de coordenação da atenção primária? Para responder a essa questão, a primeira parte do texto apresenta um panorama geral das principais formas de integração encontradas. A segunda traz uma sistematização teórico-metodológica acerca da avaliação e do monitoramento da APS com foco nos atributos relacionados com a coordenação, integralidade e continuidade do cuidado para subsidiar a apresentação de um modelo explicativo da problemática da fragmentação.

Embora sejam múltiplos os desafios que envolvem o estabelecimento e a aplicação de um sistema de monitoramento e avaliação da atenção primária, 5 é necessário caminharmos na direção de instrumentos que possam ser ao mesmo tempo abrangentes e operativos. Um estranho paradoxo parece ocorrer, criando um distanciamento entre essa necessidade e a produção de conhecimentos, com uma proliferação exagerada de conceitos, atributos e metodologias que facilitam confusões e dificultam sínteses.

Ao considerar a integração como uma categoria chave no âmbito da política de renovação da APS, esse modelo pretende mostrar a importância de distinguir e articular três níveis de análise para orientar na seleção de indicadores, possibilitando uma visão abrangente e sintética do desempenho dos sistemas de serviços de saúde.

\section{Métodos}

O estudo foi realizado no âmbito de um projeto de cooperação da Comissão Européia para promover a coesão social na América Latina através do fortalecimento de políticas públicas e da capacidade institucional de gestão denominado Eurosocial. Fez parte da fase preparatória do subprojeto "Fortalecimiento de la Integración de la Atención Primaria con otros Niveles de Atención", que envolveu um consórcio de instituições de ensino e pesquisa européias e latinoamericanas. ${ }^{6}$ Foi realizado de março a junho de 2007, através de revisão sistemática e análise de documentos (dossiês) elaborados pelos países participantes a partir de roteiro pré-estabelecido.

A divisão geopolítica do continente latino-americano, utilizada pela Organização Panamericana da Saúde compreende as seguintes sub-regiões: América Central ou Istmo Centro americano (Belice, Costa Rica, El Salvador, Guatemala, Honduras, Nicarágua, Panamá), Região Andina (Bolívia, Colômbia, Equador, Peru, Venezuela), Cone Sul (Argentina, Chile, Paraguay, Uruguay), México e Brasil. Os países participantes que enviaram o 
dossiê solicitado foram: Argentina, Brasil, Chile, Colômbia, Costa Rica, Paraguai, Uruguai e Venezuela. Portanto, a coleta de informações secundárias a partir desse instrumento cobre, pelo menos, dois países de cada uma das sub-regiões, com exceção do México. A análise desse material foi complementada com revisão bibliográfica para controlar o viés relacionado com fontes oficiais provenientes apenas da gestão nacional.

Realizou-se busca eletrônica na Biblioteca Virtual em Saúde (BVS), que compreende as plataformas Lilacs, Medline, Cochrane Library, Scielo, Pubmed, além de sites de instituições internacionais. As palavras chaves foram "integrated primary care", "integrated delivery networks" "integrated care pathways" "coordination", "linking levels of care" "outpatient referral", "atención primaria en salud" "regionalización", "niveles de cuidados/atención”, “coordinación”, "integración”, "referência", "contrarreferencia". Foram selecionados documentos que apresentassem comparações internacionais, revisões sistemáticas, posições decorrentes de consensos ou fóruns nacionais, relatos de experiências, pesquisas ou outras produções acadêmicas. Por essa razão, há no texto informações adicionais de países que não participaram do projeto, como é o caso da Nicarágua e de El Salvador, bem como, diferenças na extensão das descrições em decorrência da produção mais abundante encontrada em alguns dos casos.

\section{Resultados}

Panorama atual da integração da APS nos sistemas de saúde na América Latina

\section{Atenção primária integrada em programas} de saúde materno-infantil

Na América Latina, há uma longa tradição de ações organizadas em programas verticais focalizados em problemas de saúde específicos, entre os quais aqueles direcionados à proteção materna e infantil. No contexto das reformas sanitárias, países como Bolívia, El Salvador e Nicarágua têm investido nesse tipo de programa, cada qual com estratégias peculiares, mas tendo em comum a garantia de atenção integral apenas para mulheres e crianças. 7,8

$\mathrm{Na}$ Bolívia se ampliou o plano básico de saúde com um pacote contendo 75 serviços centrados nas principais causas de mortalidade. Esses serviços não requerem nenhum aporte financeiro dos usuários e são financiados por municípios com recursos transferidos pelo governo federal. Para garantir sua oferta tem se buscado a participação de Organizações Não Governamentais (ONGs), igrejas, prestadores privados e diversas caixas de saúde que operam no país. A partir dos resultados obtidos com esta medida foi desenhado o Seguro Universal Materno Infantil (SUM), com o propósito de superar as barreiras econômicas de acesso aos serviços enfrentadas por mães e menores de cinco anos. 7,9

Em El Salvador, se tem privilegiado a contratação de ONGs para atuação no Programa de Servicios Essenciales de Salud y Nutrición (SESYN). A estratégia se funda na concentração dos subsidios públicos e na prestação de um pacote definido de atenção integral à saúde materno-infantil, que inclui ações preventivas e curativas. Foram selecionadas quatro ONGs que recebem recursos e financiam uma rede de atenção composta por promotores de saúde e equipes itinerantes, com capacidade média de resolução e referência de casos às unidades de saúde (postos) vinculadas ao Ministério da Saúde.?

$\mathrm{Na}$ Nicarágua, o governo criou o Fondo de Maternidad e Infancia Segura (FONMAT), para financiar a oferta de um pacote de serviços para a atenção à gravidez, parto, puerpério e do recémnascido até um ano de idade. Essa política recebe recursos externos do Banco Interamericano de Desenvolvimento e o programa é operado na rede de centros e postos de saúde do Ministério da Saúde. Contam com o apoio de programas de atenção primária financiados por organismos internacionais e, também, com os Sistemas Locales de Atención Integral de Salud (SILAS) localizados em comunidades beneficiadas por este recurso. Os SILAS difundem informação, promovem práticas saudáveis e prestam serviços básicos de saúde. 7,8,10

O governo central tem apoiado os centros e postos de saúde que desenvolvem ações com base na rede de voluntariado e, o Ministério da Saúde apóia financeiramente as casas maternas administradas por governos locais e pela sociedade civil, que albergam mulheres de zonas rurais antes e depois do parto.

No pólo oposto dessa situação, onde predomina uma concepção integrada, porém focalizada em grupos prioritários, a APS surge como porta de entrada e eixo estruturante de sistemas públicos de saúde. Os sistemas com características universais estabelecidos na Costa Rica, Chile e Brasil, são os que apontam maior tendência nesta direção.

\section{A atenção primária de saúde como porta de entrada e eixo estruturante do sistema público de saúde}

$\mathrm{Na}$ Costa Rica a reorganização da atenção primária é referida como um elemento central das 
reformas mais recentes. Na reorientação do sistema, houve a transferência dos serviços de atenção primária do Ministério da Saúde para a Caja Costarricense de Seguridad Social (CCSSS) com a constituição de Equipes Básicas de Atenção Integral à Saúde (EBAIS), compostas por um médico generalista, um auxiliar de enfermagem e um assistente técnico de atenção primária em saúde. ${ }^{7}$

As EBAIS constituem a porta de entrada de um sistema desenhado a partir de um processo de territorialização com uma hierarquização dos serviços. É a partir deste primeiro nível que se identificam necessidades com encaminhamento, quando necessário, aos serviços especializados de referência e, no caso de internação, aos hospitais da área. Todos os serviços de atenção primária da CCSSS são ofertados pelas EBAIS, com exceção das emergências. 11 Apesar da existência de uma normatividade legal e organizacional são percebidas fragilidades na constituição da rede de serviços, tanto do ponto de vista da definição de competências, quanto em relação à escassez de instrumentos de planejamento e gestão que facilitem a interação e a comunicação. 12

O Chile possui um sistema de saúde cuja trajetória foi marcada por mudanças radicais, passando de um movimento de construção de um serviço nacional durante a década de 1950, para uma política de privatização após o golpe militar. Atualmente tem uma configuração mista: o sistema público, representado pelo Fondo Nacional de Salud (FONASA) (cobertura de $68 \%$ da população) e o privado constituído pelas Instituciones de Salud Previsionales (ISAPRES) (22\%). 13

O FONASA está organizado sob a forma de redes e o acesso aos serviços de maior complexidade inicia a partir da APS. Vem sendo estimulado que as unidades de um município e os demais estabelecimentos públicos e privados conveniados possam colaborar complementando ações. No âmbito dos contratos de gestão, um dos compromissos firmados entre o Ministério da Saúde e os prestadores é a constituição de um comitê de integração da rede assistencial, composto pelos diretores dos serviços. Este é um dos mecanismos considerados estratégicos para fortalecer a integração da atenção primária aos demais níveis do sistema. ${ }^{11}$

Em 1995, entrou em vigor uma lei que trata do "Acceso Universal para Prestaciones Integrales y Garantias Explícitas" (Plano AUGE) que visa assegurar a operacionalização concreta de direitos a serem exigidos nos dois subsistemas. Essas garantias cobrem problemas prioritários para os quais estão definidos parâmetros de acesso, oportunidade, qualidade e proteção financeira, sendo considerado promissor tanto para a regulação como para melhorar a problemática da coordenação em sistemas duais, onde coexistem um sistema público e privado. 13

No Brasil, com a criação do Sistema Único de Saúde (SUS), o acesso universal e a integralidade foram garantidos no plano legal. No entanto, a ausência de investimentos compatíveis com a ampliação da cobertura, favoreceu o crescimento do segmento suplementar que cobre trabalhadores de setores mais dinâmicos da economia e a população dos estratos de renda mais alto, com a existência de um mix público-privado no financiamento, prestação e utilização dos serviços. ${ }^{13}$

O processo de descentralização do SUS foi marcado por uma fragilidade da instância estadual na regionalização dos serviços e uma atuação atomizada dos municípios, o que determinou barreiras de acesso entre os sistemas municipais. Para enfrentar esta realidade, o governo federal introduziu mecanismos normativos para a institucionalização de redes de atenção regionalizadas com fortalecimento de instâncias de negociação e articulação nesse âmbito para melhorar o acesso aos níveis secundário e terciário do SUS. 14

Desde o final dos anos de 1990, a Estratégia de Saúde da Família (ESF) vem sendo considerada prioritária para potencializar a reorientação do modelo de atenção no SUS.15 Segundo a política nacional de atenção básica, cada equipe de saúde da família deve se responsabilizar em média por 3000 habitantes, sendo composta por um médico generalista, um enfermeiro, dois auxiliares de enfermagem e agentes comunitários de saúde. Estão associadas a equipes de saúde bucal, também responsáveis pelo atendimento de uma população definida. 16 Diversos estudos têm demonstrado um aumento importante da cobertura da ESF com efeitos positivos no desempenho do sistema, ${ }^{16-18}$ mas ainda são frágeis os mecanismos de referência entre níveis de atenção, o que compromete o princípio da integralidade. 18,19

Alguns municípios enfrentaram o desafio de superar os efeitos da fragmentação na rede através de um conjunto diversificado de iniciativas: Consórcios intermunicipais, 20,21 estruturação de policlínicas de especialidades, centrais de marcação (consultas especializadas, exames e de internações), 22 integração dos serviços por meio de tecnologias de informação com prontuário compartilhado, fortalecimento de estruturas regulatórias, descentralização de funções para o nível local e estabelecimento de fluxos definidos entre a atenção básica e o pronto-atendimento. 23 
APS em experiências municipais em

\section{sistemas baseados em seguros}

Na Colômbia foi criado, em 1993, um Sistema General de Seguridad Social en Salud (SGSSS) visando aumentar a cobertura através de dois Planos Obrigatórios de Saúde (POS): um para o regime contributivo e outro, mais restrito, para o subsidiado. Baseada em modalidade de seguros, a reforma colombiana não incluiu uma política nacional para atenção primária. As Entidades Promotoras de Saúde (EPS) são as operadoras responsáveis pela prestação de serviços através de um conjunto de hospitais, consultórios e centros de saúde. ${ }^{24}$

O governo de Bogotá desenvolveu um programa intitulado Salud a su Hogar baseado na APS, com a finalidade de avançar na garantia do direito a saúde, apesar das restrições impostas pela lógica do SGSSS. Esse programa está direcionado para cobrir famílias do estrato 1 e 2 do plano de asseguramento, e se apóia num enfoque familiar, comunitário e interdisciplinar. Existe a preocupação de se desenvolver mecanismos (contratos) que favoreçam a integração entre assegurador e prestador, assim como articular - Plan de Salud Obligatorio (POS) e o Plan de Atención Básica (PAB), programa de cobertura universal centrado em ações de promoção e prevenção, para garantir a integralidade e uma ação intersetorial em territórios específicos.7

$\mathrm{Na}$ Argentina, o sistema é formado por três subsetores: o sistema público operado nos três níveis de governo, nacional, provincial e municipal; o seguro social e de saúde, de afiliação obrigatória e composto pelas Obras Sociales; e o subsetor privado, com empresas de seguro, de afiliação voluntária. 11

A partir de 1997, uma série de medidas foi implementada estabelecendo um Plano Médico Obrigatório (PMO) a ser oferecido tanto pelas Obras Sociales quanto por seguradoras privadas, com incentivo à competição entre esses agentes e liberdade de escolha para os beneficiários. As Obras Sociales são responsáveis pela cobertura de $47 \%$ da população, mas em geral não possuem serviços próprios, garantindo a prestação do cuidado por meio de serviços privados ou de hospitais públicos. Não houve uma migração importante de pacientes entre as Obras Sociales, mas o PMO parece ter favorecido melhorias nas coberturas com o fortalecimento de redes de atenção primária e centros orientados para a medicina de família. 25

O setor público presta serviços através de uma rede própria e o Ministério da Saúde tem atuado na direção de um planejamento consensual com as províncias, estímulo a políticas de atenção primária com programas focalizados (distribuição de medicamentos, saúde materno-infantil - Plano Nascer e formação de médicos comunitários para ações de promoção e prevenção em nível local). ${ }^{11}$ Mas, o sistema continua essencialmente voltado para a atenção médica curativa e centrado na instituição hospitalar, sendo que as iniciativas mais relevantes na direção de práticas voltadas para a integração da APS vêm sendo realizadas em âmbito local, como é o caso, por exemplo, do município de Rosário. ${ }^{26}$

As Tabelas 1, 2 e 3 apresentam uma síntese das principais características dos sistemas analisados por tipologia das modalidades de integração da APS que foram encontradas.

A relação da integração com os atributos da APS: conceitos, matrizes e as contribuições de um modelo explicativo acerca da fragmentação dos serviços

\section{Sobre os atributos e seus conceitos}

A importância de definir atributos específicos à APS e discutir sua integração ao sistema para que possa exercer um papel de coordenação foi se tornando evidente com a avaliação do desempenho dos serviços, que mostrou a relação entre a qualidade da estrutura e do processo de cuidado para a obtenção de resultados. Para Starfield, 27 os atributos que seriam específicos da APS são: acesso, porta de entrada, vínculo (longitudinality), elenco integral de serviços (comprehensiveness), coordenação (ou integração dos serviços), enfoque familiar e a orientação para a comunidade. Seus trabalhos permitiram a criação de um instrumento para avaliá-los, conhecido como Primary Care Assessment Tool (PCATool), validado e utilizado em diversos estudos no Brasil.28,29

No entanto, há uma multiplicidade de conceitos que facilitam confusões, gerando dificuldades para operacionalizar instrumentos ágeis e mais próximos das necessidades de gestores e usuários. Ao realizar uma revisão sistemática em torno do atributo conhecido como "longitudinality" (vínculo longitudinal), Cunha ${ }^{30}$ encontrou uma proliferação de significados, alguns muito próximos à noção de continuidade, propondo sua avaliação através de um roteiro guia bastante prático e sintético.

Em nosso meio, a integralidade tem sido foco de inúmeras discussões com significados que vão desde um princípio ético que deve orientar as relações sociais no campo da saúde, até um objetivo cujo alcance necessita ser medido. Um conjunto de pesquisas já realizadas permite sugerir sua operacionalização em torno do caráter completo da 
Atenção primária à saúde seletiva em programas de saúde materno-infantil: características do sistema de saúde.

\begin{tabular}{|c|c|c|c|}
\hline Características/país & Bolívia & El Salvador & Nicarágua \\
\hline Tipo de Sistema & $\begin{array}{l}\text { Público (Ministério da } \\
\text { Saúde) } \\
\text { Seguro Social (vários) } \\
\text { Privado }\end{array}$ & $\begin{array}{l}\text { Público (Ministério da } \\
\text { Saúde) } \\
\text { Seguro Social; Privado }\end{array}$ & $\begin{array}{l}\text { Público (Ministério da Saúde) } \\
\text { Seguro Social: Instituto } \\
\text { Nicaraguense de Seguridad } \\
\text { Social (INSS); Seguro Privado }\end{array}$ \\
\hline Acesso/Cobertura & $\begin{array}{l}\text { Segmentado } \\
\text { Seletivo e focalizado } \\
\text { Seguro Universal } \\
\text { Materno Infantil }\end{array}$ & $\begin{array}{l}\text { Segmentado } \\
\text { Seletivo e focalizado } \\
\text { Programa de Servicios } \\
\text { Esenciales de Salud y } \\
\text { Nutrición (SESYN) }\end{array}$ & $\begin{array}{l}\text { Segmentado } \\
\text { Seletivo e focalizado } \\
\text { Fondo de Maternidad y } \\
\text { Infancia Segura(FONMAT) }\end{array}$ \\
\hline Financiamento & $\begin{array}{l}\text { Impostos } \\
\text { Contribuições - seguro } \\
\text { social e setor público } \\
\text { Ajuda Internacional } \\
\text { (ONGs) } \\
\text { Pagamentos diretos } \\
\text { Seguro privado }\end{array}$ & $\begin{array}{l}\text { Impostos } \\
\text { Contribuições - seguro } \\
\text { social } \\
\text { Pagamentos diretos } \\
\text { Ajuda Internacional }\end{array}$ & $\begin{array}{l}\text { Impostos } \\
\text { Contribuições INSS } \\
\text { Pagamentos diretos } \\
\text { Subsídios cruzados do setor } \\
\text { público ao INSS }\end{array}$ \\
\hline Regulação/Gestão & $\begin{array}{l}\text { Ministério da Saúde } \\
\text { Instituto Nacional de } \\
\text { Saúde }\end{array}$ & Ministério da Saúde & Ministério da Saúde \\
\hline $\begin{array}{l}\text { Gestão, } \\
\text { organização e } \\
\text { iniciativas de } \\
\text { integração da APS }\end{array}$ & $\begin{array}{l}\text { Múltiplos prestadores: } \\
\text { ONGs, seguros sociais, } \\
\text { igrejas, prestadores } \\
\text { privados; } \\
\text { Pacote Básico de ações } \\
\text { de promoção, prevenção } \\
\text { e cura para mulheres } \\
\text { grávidas e crianças até } \\
\text { cinco anos de idade }\end{array}$ & $\begin{array}{l}\text { Ministério da Saúde } \\
\text { contrata ONGs que se } \\
\text { responsabilizam por } \\
\text { rede composta por } \\
\text { promotores de saúde e } \\
\text { Equipes Itinerantes de } \\
\text { Saúde. } \\
\text { Pacote básico de ações } \\
\text { para mulheres e crianças }\end{array}$ & $\begin{array}{l}\text { INSS com rede própria } \\
\text { Empresas Médicas Provisionais } \\
\text { públicas e privadas; } \\
\text { Centros de saúde de atenção } \\
\text { primária com e sem médicos; } \\
\text { Pacotes básicos priorizam } \\
\text { mulheres e crianças; } \\
\text { Empresas Médicas Provisionais } \\
\text { e ONGs são prestadores de } \\
\text { serviços; } \\
\text { Sistemas locales de atención } \\
\text { integral a la salud (SILAIS) }\end{array}$ \\
\hline
\end{tabular}

Fonte: Elaboração própria a partir de Mesa-Lago9; Herrera10; Fleury et al.7; Flores.8

atenção, o que pode ser avaliado tanto no plano individual como no coletivo. 31,32

Para Gervas et al.,33 a capacidade de resposta da APS implicaria em selecionar e recomendar os melhores cursos de ação através de quatro funções: prevenção, resolução (curar, conter, cuidar, ajudar, consolar), derivação (filtro) e coordenação (continuidade assistencial). Portanto, a coordenação se expressa e é vivenciada pelos usuários através da garantia de continuidade assistencial e tem, pelo menos, três dimensões passíveis de avaliação e intervenção: a relação profissional/usuário (vínculo longitudinal), o uso de informação e a gestão por diferentes profissionais das respostas às necessidades dos pacientes (protocolos clínicos para doenças crônicas). 34 
Atenção primária à saúde como porta de entrada e eixo estruturante do sistema público: características dos sistemas de saúde.

\begin{tabular}{|c|c|c|c|}
\hline Características/país & Brasil & Chile & Costa Rica \\
\hline Tipo de Sistema & $\begin{array}{l}\text { Sistema público - Sistema } \\
\text { Único de Saúde (SUS) } \\
\text { Segmento suplementar }\end{array}$ & $\begin{array}{l}\text { Sistema Nacional de } \\
\text { Servicios de Salud (SNSS) } \\
\text { Seguro público de saúde } \\
\text { (FONASA) } \\
\text { Seguros Privados } \\
\text { (ISAPRES) }\end{array}$ & $\begin{array}{l}\text { Sistema público - Caja } \\
\text { Costarricense del Seguro Social } \\
\text { (CCSS); Seguros Privados }\end{array}$ \\
\hline Acesso/Cobertura & $\begin{array}{l}\text { Universal } \\
\text { Dupla cobertura, } \\
\text { segundo renda ou } \\
\text { trabalho (público + } \\
\text { privado) }\end{array}$ & $\begin{array}{l}\text { Universal } \\
\text { Capacidade de } \\
\text { pagamento - vinculação } \\
\text { ao tipo de plano público } \\
\text { e privado }\end{array}$ & $\begin{array}{l}\text { Universal } \\
\text { Contribuições e mecanismos de } \\
\text { solidariedade para garantia de } \\
\text { benefícios }\end{array}$ \\
\hline Financiamento & $\begin{array}{l}\text { Recursos fiscais (União, } \\
\text { Estados, municípios) } \\
\text { Seguros privados } \\
\text { Pagamento direto }\end{array}$ & $\begin{array}{l}\text { Contribuições (público, } \\
\text { privado) } \\
\text { Impostos } \\
\text { Copagamentos } \\
\text { Pagamento direto }\end{array}$ & $\begin{array}{l}\text { Seguro Público: Contribuições } \\
\text { sociais específicas } \\
\text { Pagamento direto }\end{array}$ \\
\hline Regulação/Gestão & $\begin{array}{l}\text { Ministério da Saúde com } \\
\text { Estados e Municípios }\end{array}$ & $\begin{array}{l}\text { Ministério da Saúde } \\
\text { SNSS - Subsecretaria de } \\
\text { Redes Assistenciais }\end{array}$ & $\begin{array}{l}\text { Ministério da Saúde } \\
\text { CCSS }\end{array}$ \\
\hline $\begin{array}{l}\text { Gestão, } \\
\text { organização e } \\
\text { iniciativas de } \\
\text { integração da APS }\end{array}$ & $\begin{array}{l}\text { Gestão municipal sob } \\
\text { regulação federal } \\
\text { Estratégia de Saúde da } \\
\text { Família: porta de entrada } \\
\text { com referência para } \\
\text { outros serviços, equipe } \\
\text { multidisciplinar, ações } \\
\text { intersetoriais } \\
\text { Consórcios municipais, } \\
\text { policlínicas de } \\
\text { especialidades, centrais } \\
\text { de marcação, definição } \\
\text { de fluxos }\end{array}$ & $\begin{array}{l}\text { MINSAL define ações de } \\
\text { APS realizada nos } \\
\text { municípios } \\
\text { Porta de entrada, } \\
\text { referência para outros } \\
\text { serviços, redes } \\
\text { assistenciais com mix } \\
\text { público-privado } \\
\text { Plano AUGE- garante } \\
\text { acesso integral para } \\
\text { problemas prioritários }\end{array}$ & $\begin{array}{l}\text { Ministério da Saúde e CCSS } \\
\text { definem ações e instrumentos } \\
\text { contratuais à implementação } \\
\text { Equipes Básicas de Atenção } \\
\text { Integral a Saúde (EBAIS), } \\
\text { referência para serviços } \\
\text { especializados e hospitalares, } \\
\text { regionalização }\end{array}$ \\
\hline
\end{tabular}

Fonte: Elaboração própria a partir de IRD Eurosocial Salud11; Mesa-Lago9; Herrera10; Conill.13

Também, é necessário estabelecer, ainda, a diferença entre a integração vertical, que é aquela que ocorre entre os níveis de cuidado (intrassistema), e a horizontal, que se dá com outros serviços, setores ou grupos sociais em nível local (intersetorial, transdisciplinar e comunitária). ${ }^{2}$ Diante de tal multiplicidade de conceitos, é interessante facilitar uma síntese a partir do resgate histórico do National Health Service (NHS), considerado paradigmático para expansão de sistemas nacionais de saúde, cuja criação assegurou um cuidado "free and comprehensive" no Reino Unido, referindo-se o termo integral ao caráter completo da cobertura (serviços ambulatoriais e hospitalares, preventivos, curativos e de reabilitação). 13

Portanto, para garantir o direito à integralidade, o cuidado necessita ser completo (tipo de ações) e, por isso, contínuo no tempo (vínculo longitudinal) e no sistema (continuidade horizontal e vertical). A coordenação é o ato oriundo de práticas locais e de 
Atenção primária à saúde em experiências municipais em sistemas baseados em seguros: características do sistema de saúde.

\begin{tabular}{|c|c|c|}
\hline Características/país & Argentina & Colômbia \\
\hline Tipo de Sistema & $\begin{array}{l}\text { Sistema público } \\
\text { Seguro social } \\
\text { Seguro privado }\end{array}$ & $\begin{array}{l}\text { Seguro Nacional de Salud } \\
\text { Regimes: contributivo e subsidiado; Seguro } \\
\text { privado }\end{array}$ \\
\hline Acesso/Cobertura & $\begin{array}{l}\text { Setor público: cobertura universal } \\
\text { Plano Médico Obrigatório (PMO): Obras } \\
\text { Sociales e seguro privado }\end{array}$ & $\begin{array}{l}\text { Plano Médico Obrigatório de Saúde (POS) } \\
\text { diferenciado por regime } \\
\text { Plano de Atenção Básica de cobertura } \\
\text { universal }\end{array}$ \\
\hline Financiamento & $\begin{array}{l}\text { Recursos fiscais } \\
\text { Contribuições } \\
\text { Pagamentos diretos }\end{array}$ & $\begin{array}{l}\text { Contribuições } \\
\text { Impostos } \\
\text { Pagamentos diretos }\end{array}$ \\
\hline Regulação/Gestão & $\begin{array}{l}\text { Governo federal } \\
\text { Províncias e governos locais responsáveis } \\
\text { pela política de APS }\end{array}$ & $\begin{array}{l}\text { Ministério de Proteção Social } \\
\text { Departamentos }\end{array}$ \\
\hline $\begin{array}{l}\text { Gestão, } \\
\text { organização e } \\
\text { iniciativas de } \\
\text { integração da APS }\end{array}$ & $\begin{array}{l}\text { APS como porta de entrada com } \\
\text { referência na rede pública em alguns } \\
\text { municípios } \\
\text { Programas federais: Plano Nacer: ações } \\
\text { focalizadas para grupo materno-infantil } \\
\text { Programa Médico Comunitário: } \\
\text { acompanhamento de famílias vulneráveis } \\
\text { e situações de risco sócio-sanitárias }\end{array}$ & $\begin{array}{l}\text { APS não se configura como política nacional, } \\
\text { iniciativas de governos locais para melhorar } \\
\text { acesso integral com ênfase na APS. } \\
\text { Empresas Promotoras de Saúde responsáveis } \\
\text { por serviços previstos no Plano de Atenção } \\
\text { Básica }\end{array}$ \\
\hline
\end{tabular}

Fonte: Elaboração própria a partir de IRD Eurosocial Salud11; Mesa-Lago9; Herrera10; Conill.13

gestão que favorece a realização desse objetivo, e tem como resultado a integração do sistema como um todo.

\section{A APS e a construção de matrizes para avaliação do desempenho dos sistemas de serviços de saúde}

Os principais modelos de matrizes de organizações internacionais ou utilizados em alguns países centrais reconhecem a interdependência de três grandes conjuntos de indicadores para os resultados dos serviços: os determinantes (ambientais, socioeconômicos e demográficos, comportamentais e biológicos), as condições de saúde da população (morbidade, estado funcional, bem-estar, mortalidade) e a estrutura (condução, financiamento, recursos). Um projeto que desenvolveu uma metodologia de avaliação para o SUS seguiu essa perspectiva, inter-relacionando esses indicadores com aqueles que se referem aos resultados, tais como, a efetividade, o acesso, a eficiência, o respeito ao direito das pessoas, a aceitabilidade, a continuidade, a adequação e a segurança. ${ }^{35}$ Muitos indicadores têm uma relação direta com os atributos anteriormente analisados e que caracterizam as funções da atenção primária.

No Canadá, em 2004, foi desenvolvido um modelo para identificar resultados que poderiam ser diretamente atribuídos às práticas de APS. ${ }^{36}$ Esse modelo assinala fatores do contexto que influenciam o desempenho destacando os produtos a serem avaliados, tanto do ponto de vista quantitativo (volume, produtividade) como qualitativo ("responsiveness", integralidade, continuidade, coordenação, comunicação interpessoal e efetividade técnica), por níveis hierárquicos e inter-relacionados.

Há, ainda, uma diferença importante entre o nível individual e o nível coletivo do conceito de 
qualidade, que necessita, também, ser considerada: no primeiro, a qualidade é percebida como adequada ao obtermos cuidado resolutivo quando dele necessitamos; no segundo, o interesse é outro, focando-se na equidade e nos custos. ${ }^{37}$ Ao formular uma matriz com indicadores de qualidade para países da Organization for Economic Cooperation and Development (OECD) (Health Care Quality Indicators - HCQI Project), o estudo de Arah et al. 38 segue uma tendência que vem predominando na construção de matrizes de avaliação. Ou seja, é necessário incorporar dados do contexto social, econômico, organizacional, mas, também, ter cuidado em identificar para fins de análise as esferas macro, meso e microssocial. ${ }^{39}$ Ao estudarmos a problemática da fragmentação dos serviços de saúde através do mapeamento apresentado, foi possível identificar variáveis e desenvolver um modelo explicativo que articula esses três níveis de análise, e que poderá ser útil na avaliação do desempenho dos serviços orientados pela APS.

Assim, em nível macrossocial ocorrem decisões políticas acerca dos direitos de acesso, coberturas, financiamento e a macrorregulação (políticas de informação, planejamento, avaliação, desenvolvimento de recursos humanos, científico e tecnológico). Em nível intermediário, nível meso ou da gestão, localiza-se um conjunto de atividades de suporte necessárias ao desempenho adequado das práticas de saúde. Nesse nível importa observar não apenas as decisões, mas principalmente a implementação de mecanismos operativos, tais como, fluxos, suporte tecnológico, formação, comunicação e informação, e regulação profissional. E, em nível microssocial, onde ocorre o cuidado, as relações interpessoais é que são importantes, com predomínio dos atributos relacionados ao acesso, à comunicação, informação e à efetividade da atenção. A Figura 1 resume esses processos por níveis e atributos.

Figura 1

Modelo lógico para avaliação de sistemas orientados pela atenção primária de saúde.

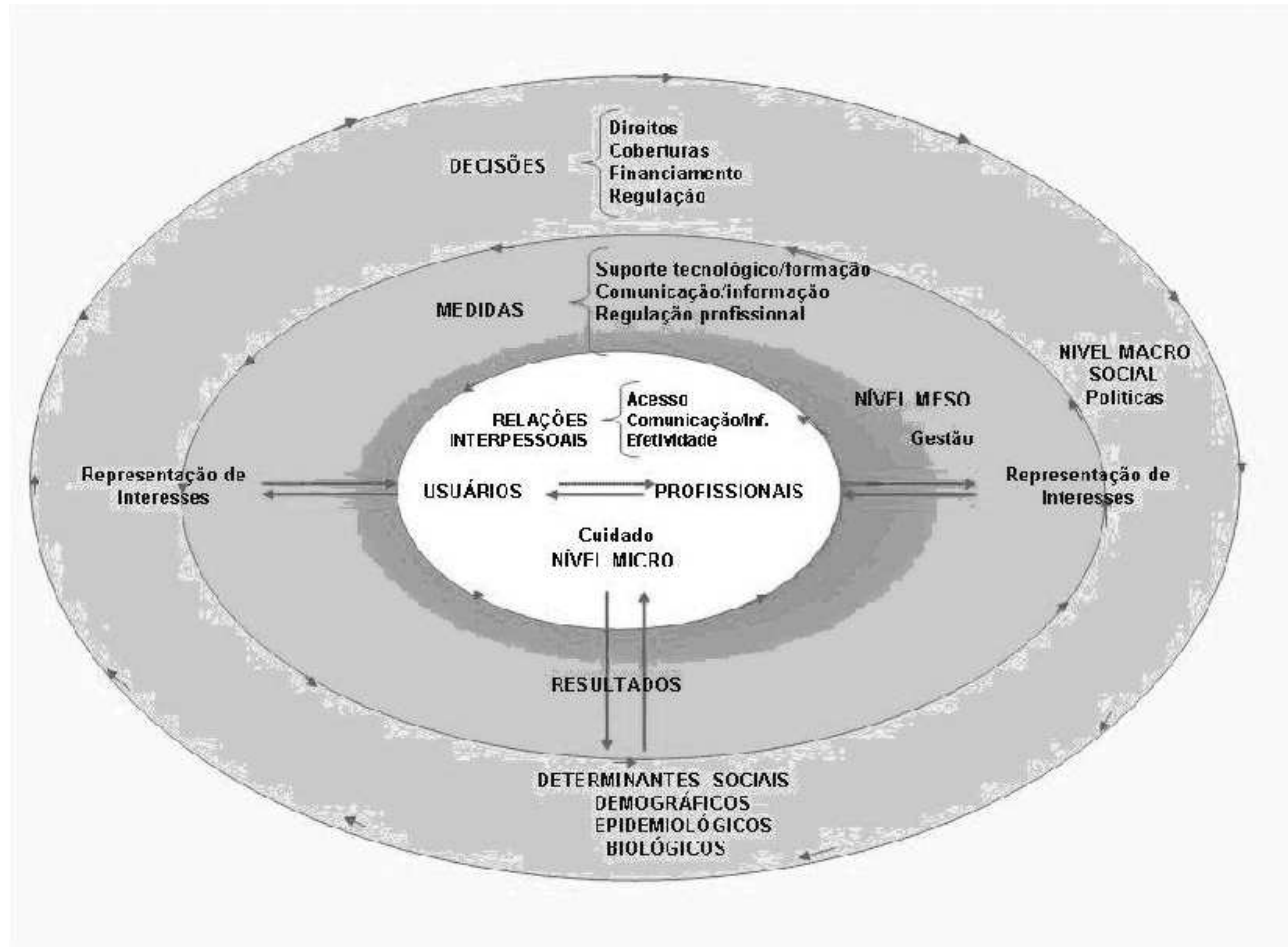

Fonte: Elaboração própria a partir de Conill e Fausto. ${ }^{2}$ 


\section{Discussão}

Embora haja uma política continental visando a renovação e o fortalecimento da APS, a situação encontrada mostra que a integração e coordenação com os demais serviços ainda é frágil. Três principais modalidades foram identificadas: atenção primária integrada por dentro dos programas de saúde materno-infantil; como porta de entrada e eixo estruturante do sistema público de saúde; e em experiências municipais em sistemas com seguros. Na primeira predomina a noção de uma coordenação entre serviços no interior de um mesmo programa; há integração das práticas de APS com serviços especializados numa perspectiva focalizada em grupos prioritários. Embora essa estratégia, conhecida como Integrated Management of Childhood Illness (IMCI), pareça ter resultados favoráveis na redução da mortalidade, haveria limites em função de uma cobertura e uma racionalidade de gestão voltada apenas para as ações da área materno-infantil. 40

No caso de sistemas públicos onde é considerada uma política de reorganização do modelo assistencial, são distintos os graus de implementação e resultados das medidas para facilitar sua integração. As principais estratégias encontradas foram: territorialização com adscrição de clientela, equipes multidisciplinares, informatização de prontuários, centrais de marcação de consultas, equipes especializadas de apoio, consórcios para atenção especializada, comitês de integração de serviços, criação de redes e estímulo à gestão regional. A terceira situação se caracteriza por iniciativas isoladas de governos municipais, que parecem ainda não ter possibilitado a aglutinação de forças sociais suficientes para alcançar modificações mais profundas nas políticas sociais e de saúde do país. ${ }^{41}$

Entretanto, é preciso considerar que a configuração de um sistema de saúde e de suas reformas é um processo complexo e dinâmico, marcado pela interação de diversos fatores que se modificam no tempo. Outras vezes, aspectos formais garantidos no plano legal não correspondem ou demoram a se expressar em mudanças práticas de direitos e coberturas, o que implica em limites para a tipologia sugerida.

Os sistemas para avaliação e monitoramento desenvolvidos em diversos países, inclusive no Brasil, possuem grandes semelhanças e reconhecem a interligação entre variáveis do contexto com as condições necessárias ao funcionamento dos serviços e seu desempenho. As categorias de avaliação mais frequentemente utilizadas visam medir acesso, efetividade, adequação, continuidade, aceitabilidade, segurança, sustentabilidade, eficiência e equidade, entre outras.

A existência atual de uma profusão semântica e metodológica tem trazido dificuldades para o campo da avaliação e para compatibilização dessas matrizes com a crescente importância das políticas de atenção primária. Por outro lado, referenciais e instrumentos dirigidos para avaliar apenas as práticas de APS podem, contraditoriamente, incentivar uma percepção desarticulada dessas ações.

A descrição e análise da integração da APS nos sistemas de saúde da América Latina mostraram que, mesmo nos casos de sistemas de base universal, há persistência de segmentação com fragmentação dos serviços. O modelo proposto permite articular essas duas categorias, que são, atualmente, centrais para a compreensão da problemática da qualidade dos serviços, de modo a facilitar uma visão abrangente do desempenho dos sistemas orientados por essa política.

Em sistemas nos quais a APS constitui uma política nacional, no acompanhamento das decisões em nível macro podem ser utilizados indicadores que informem sobre a evolução do financiamento público, da proporção de gastos com a atenção primária, ou de profissionais de APS em relação ao conjunto de recursos humanos, especialmente dos médicos. Em nível meso ou da gestão, a obtenção de dados sobre os tempos de espera para acesso aos serviços especializados e sobre a satisfação de usuários e profissionais, talvez possam revelar de forma rápida e sintética os resultados de medidas para facilitar a integração. Em nível local, o acompanhamento do tempo de espera, da gama de serviços, seu uso regular, a satisfação e alguns marcadores selecionados da efetividade das ações, representam exemplos de informações que podem vir a completar um panorama a partir do modelo sugerido.

Esse referencial também permite identificar a existência ou não de uma coerência entre os diversos locus das ações, uma vez que é difícil sustentar cuidados integrais em nível local, sem que ocorram medidas e decisões compatíveis nas demais esferas de governo. Mas o verdadeiro desafio na montagem de sistemas de avaliação é de que possam servir para impulsionar mudanças que levem a melhores resultados dos serviços. Para isso, é importante ter em mente que os indicadores e as matrizes propostas trazem informações de processos situados em níveis sociais que apresentam dinâmicas profundamente diversas. Uma das vantagens do modelo desenvolvido é permitir a separação clara desses níveis, 
pois as intervenções que se farão necessárias implicam em abordagens e interações entre atores sociais muito distintos.

A questão da credibilidade da APS tem se constituído num entrave na implementação dessa política pela relativa fragilidade deste nível de atenção frente à intenção de fazê-lo assumir uma função estratégica. Conforme mostramos, todos os atributos considerados específicos da atenção primária estão inter-relacionados. Por essa razão um sistema

\section{Referências}

1. Saltman R. Convergence versus social embeddedness, debating the future direction of health care systems, Eur J Public Health. 1997; 7: 449-53.

2. Conill EM, Fausto MCR. Análisis de la problemática de la integración APS em el contexto actual: causas que inciden en la fragmentación de servicios y sus efectos en La cohesión social. Proyecto EUROsociAL Salud, Intercambio "Fortalecimiento de la Integración de la Atención Primaria com otros Niveles de Atención" Documento técnico. Rio de Janeiro: IRD, 2007 [2010 Abr 10]. Disponível em: http://www.eurosocialsalud.eu/docs/p4_1_1_1/MzI=

3. Organização Panamericana de Saúde. Renovação da atenção primária em saúde nas Américas. Documento de posicionamento da Organização Pan-Americana de saúde/OMS. Brasília, DF: OMS; 2005.

4. Haggerty JL, Yavich N, Báscolo EP, Grupo de Consenso sobre un Marco de Evaluación de la Atención Primaria en América Latina. Un marco de evaluación de la atención primaria de salud en América Latina. Rev Panam Salud Publica. 2009; 26: 377-84.

5. Felisberto E, Freese E, Alves CKA, Bezerra LKA, Samico I. Política de monitoramento e avaliação da atenção básica no Brasil de 2003 a 2006: contextualizando sua implantação e efeitos. Rev Bras Saude Mater Infant. 2009; 9: 33957. Disponível em: http://www.scielo.br/pdf/rbsmi/ v9n3/13.pdf

6. IRD EUROsociAL Salud [Internet]. Madrid (ES): IRD [acesso em 2010 Mar 10]. Proyecto Eurosocial Salud. Disponível http://www.eurosocialsalud.eu/proyecto/p1_1_5

7. Fleury S, Belmartino S, Baus E. Reshaping health care in Latin America. A comparative analysis of health care reform in Argentina, Brasil and México [Internet]. Canadá, International Development Research Center, 2000 [acesso 2007 Mai 28]; [292p]. Disponível em: http://www.idrc.ca/en/ev-9421-201-1-DO_TOPIC.html .

8. Flores W. Equidad y reformas del sector salud en America Latina y el Caribe: experiencias y lecciones aprendidas. Informe Sociedad Internacional por la equidad em Salud [Internet]. Versión para discussión. Mayo de 2005 [acesso 2007 Mai 21]. Disponível em: http://www.revmed.unal.edu.co/equidad/doc/Foro/RSS\%20 y\%20equidad-5\%20MAYO\%5B2\%675D.pdf . adequadamente coordenado e integrado, com um bom desempenho na continuidade do cuidado, terá reflexos positivos na procura dos serviços como porta de entrada, no vínculo, na integralidade, e na credibilidade da APS. A procura por um marco abrangente para a avaliação de sua implementação, que considere as distintas modalidades desta implementação em cada país, levando em conta níveis inter-relacionados de análise, poderá contribuir para o avanço dessa política em nosso continente.
9. Mesa-Lago C. Las reformas de salud en América Latina y el Caribe: su impacto en los principios de la seguridad social. Documentos de projectos [Internet]. Santiago del Chile: CEPAL; Deciembre del 2005 [acesso 2007 Mai 26]. Disponível em: http://www.eclac.cl/publicaciones/xml/8/ 24058/LCW63 ReformasSalud ALC Indice.pdf

10. Herrera AR. Las reformas de salud y nuevos modelos de atención primaria en America Central [Internet]. Santiago del Chile: CEPAL; 2006 [acesso 2007 Mai 25]. Disponível em: http://www.eclac.cl/publicaciones/xml/7/26117/ lc12524e.pdf

11. IRD EUROsociAL Salud. Dossiers nacionales de atención primaria en salud y integración con otros niveles de atención. Intercambio III. 02 de janeiro de 2007. EuropeAid, Fundación para la Cooperación y Salud Internacional Carlos III. Rio de Janeiro: Ensp/Fiocruz; 2007.

12. Castañeda AV. El hospital y los desafíos para la integración a la red de servicios: El caso del Hospital México, Costa Rica. In: Seminario Inter-Tematico Eurosocial Salud: atencion primaria de la salud e integracion de niveles de atención. 24 a 26 de setembro de 2007. Escola Nacional de Saúde Pública Sérgio Arouca. Rio de Janeiro: ENSP/Fiocruz; 2007.

13. Conill EM. Sistemas comparados de saúde. In: Campos GWS, Minayo MCS, Akerman M, Drumond Junior M, Carvalho YM, organizadores. Tratado de Saúde Coletiva. São Paulo/Rio de Janeiro: HUCITEC/Fiocruz; 2006. p. 563-614.

14. Brasil. Ministério da Saúde. Secretaria Executiva Departamento de Apoio à Descentralização. CoordenaçãoGeral de Apoio à Gestão Descentralizada. Diretrizes operacionais dos pactos pela vida, em defesa do SUS e de gestão. Brasília, DF; 2006. Série A. Normas e Manuais Técnicos.

15. Brasil. Ministério da Saúde. Secretaria de Atenção Básica. Departamento de Atenção Básica. Saúde da família no Brasil: uma análise de indicadores selecionados, 19982004. Brasília, DF; 2006.

16. Brasil. Ministério da Saúde. Secretaria de Atenção à Saúde. Departamento de Atenção Básica. Política nacional de atenção básica. Brasília, DF; 2006. Serie Pactos pela Saúde 2006; 4 . 
17. Macinko J, Guanais FC, Souza MF. An Evaluation of the Impact of the Family Health Program on Infant Mortality in Brazil, 1990-2002. J Epidemiol Community Health. 2006; 60: 13-9.

18. Escorel S, Giovanella L, Mendonça MHM, Senna MCM. O Programa de saúde da família e a construção de um novo modelo para a atenção básica no Brasil. Rev Panam Salud Publica. 2007; 21: 164-76.

19. Conill EM. Políticas de atenção primária e reformas sanitárias: discutindo a avaliação a partir da análise do programa saúde da família em Florianópolis, Santa Catarina, Brasil, 1994-2000. Cad Saúde Pública. 2002; 18 (Supl.): 191-202.

20. Neves LA, Ribeiro JM. Consórcio em saúde: estudo de caso exitoso. Cad Saúde Pública. 2006; 22: 2207-17. Disponível em: http://www.scielo.br/pdf/csp/v22n10/20.pdf .

21. Guimarães L, Giovanella L. Cooperação intergovernamental: os consórcios em saúde de Mato Grosso. Saúde Debate. 2004; 28: 149-58.

22. Senna MCM, Cohen MM. Modelo assistencial e estratégia saúde da família no nível local: análise de uma experiência Ciên Saúde Colet. 2002; 7: 523-35. Disponível em: http://www.scielo.br/pdf/csc/v7n3/13029.pdf .

23. Prefeitura Municipal de Belo Horizonte (Minas Gerais/BR). Secretaria Municipal de Saúde; Secretaria de Coordenação das Políticas Sociais. BH-Vida: saúde integral. Diretrizes para o avanço e articulação do processo assistencial na SMSA para o período 2003-2004. Belo Horizonte, MG; 2003.

24. Gómez-Camelo D. Análisis comparado de los sistemas de salud de la región Andina y el Caribe. Rev Salud Publica 2005; 7: 305-16. Disponível em: http://www.scielosp.org/ pdf/rsap/v7n3/v7n3a06.pdf.

25. Findling L, Arrunãda M, Klimovsky E. Desregulación y equidad: el processo de reconversión de Obras Sociales en Argentina. Cad Saúde Pública. 2002; 18: 1077-88. Disponível

em: http://www.scielo.br/pdf/csp/v18n4/10187.pdf .

26. Rovere M. La salud en el municipio de Rosário: aportes a la construcción de una gobernabilidad democrática. In: Experiencia Rosário: políticas para la gobernabilidad. Rosário: Municipalidad de Rosario/PNUD; 2006. p. $107-$ 164 [acesso 2010 Abr 02]. Disponível em: http://www.undp.org.ar/docs/Libros y Publicaciones/Gobe rnabilidad.pdf

27. Starfield B. Atenção primária: equilíbrio entre necessidades de saúde, serviços e tecnologia. 2 ed. Brasília: UNESCO/Ministério da Saúde; 2004.

28. Almeida C, Macinko J. Validação de uma metodologia de avaliação rápida das características organizacionais e do desempenho dos serviços de atenção básica do Sistema Único de Saúde (SUS) em nível local. Brasília: OPAS; 2006

29. Elias PE, Ferreira CW, Alves MCG, Cohn A, Kishima V, Escrivão Júnior A, Gomes A, Bousquat A. Atenção básica em Saúde: comparação entre PSF e UBS por extrato de exclusão social no município de São Paulo. Ciên Saúde Colet. 2006; 11: 633-41. Disponível em: http://www.scielo.br/pdf/csc/v11n3/30979.pdf .
30. Cunha EM.Vínculo longitudinal na atenção primária: avaliando os modelos assistenciais do SUS [tese] Rio de Janeiro: Programa de Pós-Graduação de Saúde Pública da Escola Nacional de Saúde Pública Sérgio Arouca. Fundação Oswaldo Cruz; 2009.

31. Conill EM. Avaliação da integralidade: conferindo sentido para os pactos na programação de metas dos sistemas municipais de saúde. Cad Saúde Pública. 2004; 20: 141723.

32. Giovanella L, Lobato L, Carvalho AL, Conill EM, Cunha EM. Sistemas municipais de saúde e a diretriz da integralidade da atenção: critérios para avaliação. Saúde Debate. 2002; 26: 37-61.

33. Gervas J, Mena OP, Mainar AS. Capacidad de respuesta de la atención primaria y buena reputación profesional, algo más que buen trabajo clínico. Med Clin (Barc). 2007; 128: 540-4. Disponível em: http://www.elsevier.es/revistas/ctl_ servlet?_f=7010\&sumarioid $=13004407 \&$ anteriores $=$ true .

34. Boerma WGW. Coordination and integration in European primary care. In: Saltman RB, Rico A, Boerma WGW, editors. Primary care in the driver's seat? Organizational reform in European primary care. Berkshire: Open University Press; 2006. European Observatory on Health Sistems and Policies Series. p 3-21. [Acesso 2010 Mar 16]. Disponível em: http://www.moh.govt.nz/notebook/ nbbooks.nsf/0/0b22ec7fc7dc93b8cc257144008053d3/\$FIL E/E87932.pdf

35. Almeida C, Szwarcwald CL, Travassos C, Viacava F, Novaes HMD, Noronha JC, Rocha JY, Silva LMV, Almeida MF, Barros M, Martins M, Ibãnes N, Ugá MA, Caetano R, Porto S, Macinko J. Projeto: Desenvolvimento de metodologia de avaliação do desempenho do sistema de saúde brasileiro (PRO-ADESS). Relatório Final. Rio de Janeiro: CICT/Fiocruz; agosto de 2003 [acesso 2010 Mar 03]. Disponível em: http://www.proadess.cict.fiocruz.br/ index $2 \mathrm{v} \cdot \mathrm{htm}$.

36. Watson DE, Broemeling A-M, Reide RJ, Black C, Centre for Health Services and Policy Research. A results-based logic model for primary health care. Vancouver, BC (Canadá): University of British Columbia; 2004 [Acesso 2010 Mar 16]. Disponível em: http://www.chspr.ubc.ca/ files/publications/2004/chspr04-19.pdf .

37. Campbell SM, Roland MO, Buetow SA. Defining quality of care. Soc Sci Med. 2000; 51: 1611-25.

38. Arah OA, Wespert GT, Hurst J, Klazinga NS. A conceptual framework for the OECD health care quality indicators project. Int J Qual Health Care. 2006; 18 (Suppl 1): 5-13.

39. Silva SF. Desafios para o aperfeiçoamento das redes de atenção à saúde no SUS no contexto da implementação do pacto pela saúde. In: Silva SF, organizador. Redes de atenção à saúde no SUS: o pacto pela saúde e redes regionalizadas de ações de serviços de saúde. Campinas: IDISA/CONASEMS; 2008. Cap III. 2. p. 117-36.

40. Bryce J, Victora CG, Habitcht JP, Black RE, Scherpbier RW, MCE-IMCI Technical Advisors. Programmatic pathways to child survival: results of a multi-country evaluation of integrated management of childhood illness. Health Policy Plan. 2005; 20 (Suppl 1): i5-i17. 
41. Romero RV, Franco JC. APS y acesso universal a los servicios de salud en lãs condiciones del SGSSS de Colombia. Anais do 11 Congresso Internacional de Saúde Pública e 8 Congresso Brasileiro de Saúde Coletiva; 21 a 25 de agosto de 2006; Rio de Janeiro: ABRASCO/WFPHA; 2006. (CD ROOM)

Recebido em 13 de abril de 2010

Versão final apresentada em 21 de setembro de 2010

Aprovado em 30 de setembro de 2010 